
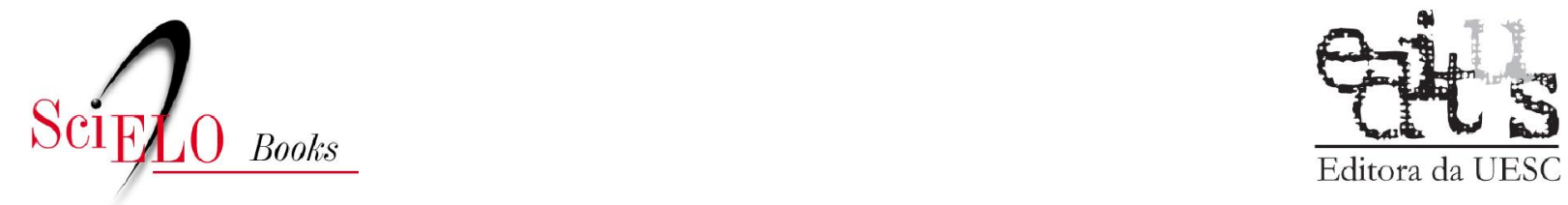

Editora da UESC

\title{
7 - Estado da arte sobre a taxonomia e filogenia de Proceratiinae
}

\author{
Rodrigo M. Feitosa
}

SciELO Books / SciELO Livros / SciELO Libros

FEITOSA, RM. Estado da arte sobre a taxonomia e filogenia de Proceratiinae. In: DELABIE, JHC., et al., orgs. As formigas poneromorfas do Brasil [online]. Ilhéus, BA: Editus, 2015, pp. 75-84. ISBN 978-85-7455-441-9. Available from SciELO Books < http://books.scielo.org $>$.

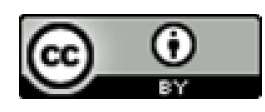

All the contents of this work, except where otherwise noted, is licensed under a Creative Commons Attribution $\underline{4.0 \text { International license. }}$

Todo o conteúdo deste trabalho, exceto quando houver ressalva, é publicado sob a licença Creative Commons Atribição 4.0.

Todo el contenido de esta obra, excepto donde se indique lo contrario, está bajo licencia de la licencia Creative Commons Reconocimento 4.0. 


\title{
Estado da arte sobre a taxonomia e filogenia de Proceratiinae
}

\author{
Rodrigo M. Feitosa
}

\section{Resumo}

Proceratiinae atualmente engloba três gêneros de formigas em duas tribos: Discothyrea e Proceratium na tribo Proceratiini e Probolomyrmex como único membro da tribo Probolomyrmecini. A subfamília está presente em todo o mundo, especialmente nas regiões tropicais, onde a maior parte de suas cerca de 140 espécies ocorre. Nas Américas, Proceratiinae apresenta cerca de 40 espécies distribuídas desde o sul do Canadá até a Argentina. No Brasil, os três gêneros da subfamília estão representados por apenas seis espécies formalmente descritas, sendo que Probolomyrmex apresenta três espécies, Discothyrea possui duas espécies e Proceratium está representado no país por uma única espécie.

Proceratiinae pode ser morfologicamente reconhecida pela seguinte combinação de caracteres: tamanho relativamente pequeno; inserções antenais total ou parcialmente expostas; olhos reduzidos a ausentes; sutura promesonotal fundida ou ausente; garras tarsais anteriores simples, nunca pectinadas ou com dentes pré-apicais; pecíolo sem tergitos laterais; e lobo jugal ausente nas asas anteriores das formas sexuadas. Embora diferentes estudos indiquem que Proceratiinae seja um membro do complexo poneroide de subfamílias, nenhum estudo filogenético foi proposto para testar suas relações internas. Estudos moleculares recentes que incluíram membros de Proceratiinae entre seus terminais parecem apontar para uma posição basal de Discothyrea que aparece como grupo -irmão de um clado formado por Probolomyrmex + Proceratium. Ainda assim, um estudo mais abrangente e que inclua um número maior de espécies se faz necessário para o estabelecimento das relações filogenéticas em Proceratiinae.

Esta subfamília é relativamente pouco representada em coleções mirmecológicas. Isso ocorre porque os indivíduos são raramente capturados com as técnicas mais tradicionais de coleta. O extrator de Winkler tem se mostrado o método mais eficiente para amostrar exemplares de Proceratiinae, embora estes sejam coletados sempre em número bastante reduzido. Esta aparente raridade se deve em grande parte ao hábito críptico das espécies e às baixas densidades populacionais das colônias, que raramente alcançam 100 indivíduos. Os ninhos são normalmente encontrados em cavidades no solo de florestas, entre as folhas da serapilheira, troncos em decomposição e sob rochas. Com relação ao hábito alimentar, as observações feitas até então sugerem uma preferência destas formigas por ovos de pequenos artrópodes de solo, em especial aracnídeos. Virtualmente nada é conhecido sobre o sistema de forrageamento das espécies proceratiíneas e suas estratégias reprodutivas.

FEITOSA, Rodrigo M. Estado da arte sobre a taxonomia e filogenia de Proceratiinae. In: DELABIE, Jacques H. C. et al. As formigas poneromorfas do Brasil. Ilhéus: Editus, 2015. p. 75-84. 
State of the art of the taxonomy and phylogeny of the subfamily Proceratiinae The subfamily Proceratiinae comprises three ant genera in two tribes: Discothyrea and Proceratium in the Proceratiini, and Probolomyrmex as the only member of the Probolomyrmecini. The subfamily is present in all regions of the world, especially in the tropics, where most of its 140 species occur. In the New World, Proceratiinae contains around 40 species distributed from southern Canada to Argentina. In Brazil, the three genera of the subfamily are represented by only six species; three species of Probolomyrmex, two of Discothyrea and a single species of Proceratium.

Proceratiinae can be morphologically recognized by the following combination of characters: size relatively small; antennal insertions totally or partially exposed; eyes strongly reduced to absent; promesonotal suture fused or absent; tarsal claws of forelegs simple, never pectinate nor possessing preapical projections; petiole without laterotergites; and jugal lobe absent in the forewings. Although the position of Proceratiinae within the poneroid subfamilies is relatively well established, a phylogenetic study to investigate the internal relations of the subfamily has never been proposed. Molecular studies that have included proceratiine species as terminals seem to indicate a basal position for the genus Discothyrea, which represents the sister group of a clade formed by Probolomyrmex + Proceratium. However, a broader study including a high number of species is needed to improve the knowledge on the phylogenetic relationships in Proceratiinae.

The subfamily is relatively poorly represented in myrmecological collections. This is due the fact that individuals are rarely captured by traditional collecting techniques. The Winkler apparatus has proved to be the most efficient method to collect specimens of Proceratiinae, although the number of individuals in the samples is always very low. This apparent rarity is mostly due the cryptic habits of the species and the relatively low size of colonies, which rarely reach 100 individuals. Nests are often found in cavities in the forest floor, in the interstices of the leaf litter, decaying logs, and under stones. Regarding the diet, the few observations made thus far suggest a preference of these ants to prey upon eggs of small soil arthropods, particularly arachnids. Virtually nothing is known about the foraging systems and the reproductive strategies of most proceratiine species.

\section{Sistemática de Proceratiinae}

Diferentes estudos recentes têm adicionado importantes informações sobre a taxonomia e filogenia das linhagens de formigas em um nível abrangente (ver Capítulo 8 deste livro por FERNÁNDEZ; LATTKE); no entanto, há ainda um déficit considerável acerca da filogenia interna de boa parte das subfamílias, tribos ou mesmo gêneros, o que é verdade também para a subfamília Proceratiinae. Até 2003, a maioria das formigas desta subfamília era considerada como pertencente à tribo Ectatommini que, juntamente com outras tribos, formavam um táxon muito abrangente e heterogêneo, a subfamília Ponerinae (BOLTON, 1994; BOLTON, 1995).

Brown (1958) apresentou a primeira proposta de classificação para Ectatommini. Neste trabalho, uma série extensiva e bem justificada de sinonímias genéricas reduziu um imenso arranjo de nomes a apenas nove gêneros (Acanthoponera, Aulacopone, Discothyrea, Ectatomma, Gnamptogenys, Heteroponera, Paraponera, Proceratium e Rhytidoponera). Neste período, o gênero Probolomyrmex era considerado membro da tribo Platythyreini.

Apesar de não ter realizado uma análise cladística, Brown (1958) comparou a morfologia de Ectatommini a de diversos outros grupos de Ponerinae, concluindo que Ectatommini apresentava os gêneros mais basais, sendo Acanthoponera o gênero que reunia o maior número de características ancestrais e do qual se derivava diretamente Heteroponera que, por sua vez, seria mais intimamente relacionado à Aulacopone, Discothyrea, Gnamptogenys, Proceratium e Rhytidoponera, enquanto Ectatomma e Paraponera formariam uma linhagem distinta. 
Em 1975, Brown publica a última parte de sua série de trabalhos sobre a sistemática de Ponerinae. Neste artigo, Brown revisa a tribo Platythyreini e comenta as relações entre Probolomyrmex e Platythyrea. Embora tenha incluído ambos na mesma tribo, Brown reconhece as diferenças marcantes entre estes gêneros, especialmente no que se refere à morfologia das larvas. Ainda assim, nos trabalhos subsequentes, a classificação de Brown para as tribos poneríneas foi mantida por diferentes autores.

Em 1994, Lattke propôs uma análise cladística para Ectatommini utilizando um grande conjunto de caracteres de morfologia externa. Neste estudo, Lattke demonstrou que Ectatommini era um grupo polifilético e propôs uma nova classificação para os gêneros que o compõem, revalidando as tribos Paraponerini, com Paraponera como único membro e Proceratiini, englobando Discothyrea e Proceratium. Os demais gêneros foram mantidos em Ectatommini (Figura 7.1A).

Os resultados de Lattke foram contestados por Keller (2000) que afirmou que a classificação proposta era filogeneticamente inconsistente, já que Ectatommini era polifilética e as análises de

Figura 7.1 - Relações entre os gêneros de Ectatommini sensu Brown (1958), com as propostas para a reclassificação da tribo segundo (A) Lattke (1994) e (B) Keller (2000). (Modificado de Feitosa, 2011)
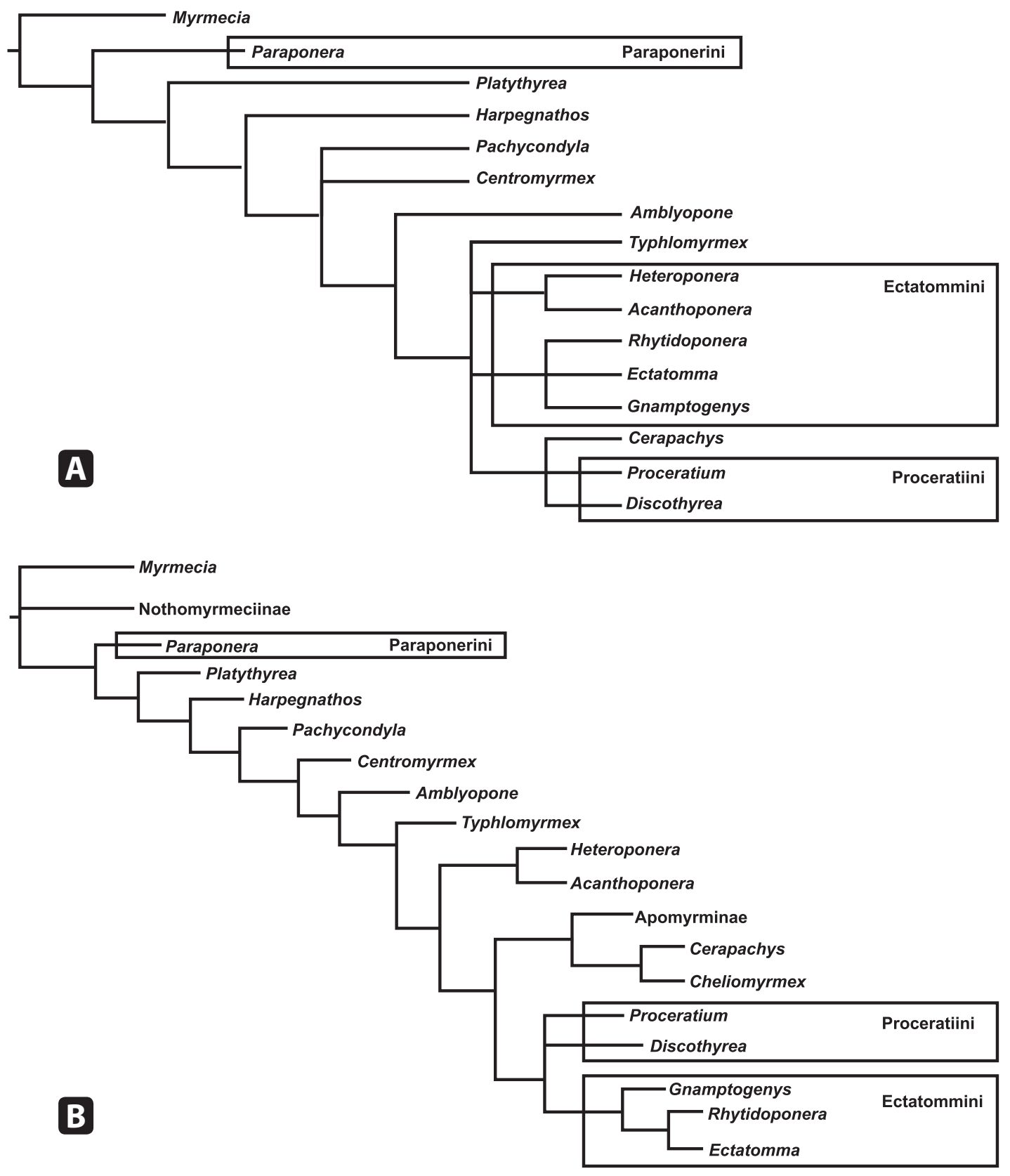
Lattke foram baseadas em propostas de classificação irrelevantes para a escolha dos grupos externos. Keller ampliou a matriz de Lattke com novos táxons e caracteres e desenvolveu uma nova análise filogenética (Figura 7.1B). Seus resultados confirmaram a distância evolutiva entre Paraponera e os demais gêneros e também a monofilia do clado formado por Heteroponera, Ectatomma e Rhytidoponera. Contudo, a relação encontrada por Lattke entre Discothyrea e Proceratium não foi demonstrada por Keller, invalidando a justificativa de Lattke para a criação da tribo Proceratiini e perpetuando a instabilidade nas classificações do grupo.

Dadas as evidências de que Ectatommini e mesmo Ponerinae constituíam agrupamentos artificiais, Bolton (2003) desmembrou boa parte das tribos reconhecidas por Brown $(1958,1975)$ e dividiu Ponerinae em seis subfamílias: Amblyoponinae, Ectatomminae, Heteroponerinae, Paraponerinae, Ponerinae e Proceratiinae. A maior parte dos gêneros que até então formavam a tribo Ectatommini agora se encontram divididos nas subfamílias Ectatomminae (Ectatomma, Gnamptogenys, Rhytidoponera e Typhlomyrmex), Heteroponerinae (Acanthoponera, Aulacopone e Heteroponera) e Proceratiinae (Discothyrea, Probolomyrmex e Proceratium). Assim, Proceratiinae aparece pela primeira vez na literatura e além dos gêneros Discothyrea e Proceratium (já tidos como filogeneticamente relacionados em trabalhos anteriores) passa a englobar o gênero Probolomyrmex, até então considerado membro da tribo Platythyreini.

No mesmo trabalho, Bolton (2003) considerou as subfamílias originadas do desmembramento de Ponerinae como membros de um agrupamento informal, o qual chamou de poneromorfas. Este termo foi aceito por algum tempo e apareceu com frequência na literatura dos anos seguintes ao trabalho de Bolton. No entanto, filogenias recentes utilizando dados moleculares demonstraram que as poneromorfas formam na verdade uma assembleia artificial, com um grupo monofilético, o complexo poneroideo (no qual se inclui Proceratiinae), excluindose as subfamílias Ectatomminae e Heteroponerinae que juntas formam o complexo ectaheteromorfo e, ao lado de Formicinae e Myrmicinae, compõem o atual clado formicoide (MOREAU et al., 2006; OUELLETTE et al., 2006; BRADY et al., 2006; RABELING et al., 2008) (Figura 7.2).

Mais recentemente, Keller (2011) publicou uma das análises filogenéticas mais abrangentes já feitas para formigas, utilizando exclusivamente caracteres morfológicos, com ênfase nos grupos incluídos no complexo poneromorfo de Bolton (2003). Apesar das grandes divergências entre este trabalho e as propostas recentes que utilizaram dados moleculares, a análise de Keller comprova a parafilia do complexo poneromorfo.

Os estudos moleculares recentes que incluíram membros de Proceratiinae entre seus terminais parecem apontar para uma posição basal de

Figura 7.2 - Sumário das relações filogenéticas entre subfamílias de formigas reconstruídas com base em dados moleculares. O tamanho dos triângulos indica a diversidade de cada subfamília (Modificado de Keller, 2011)

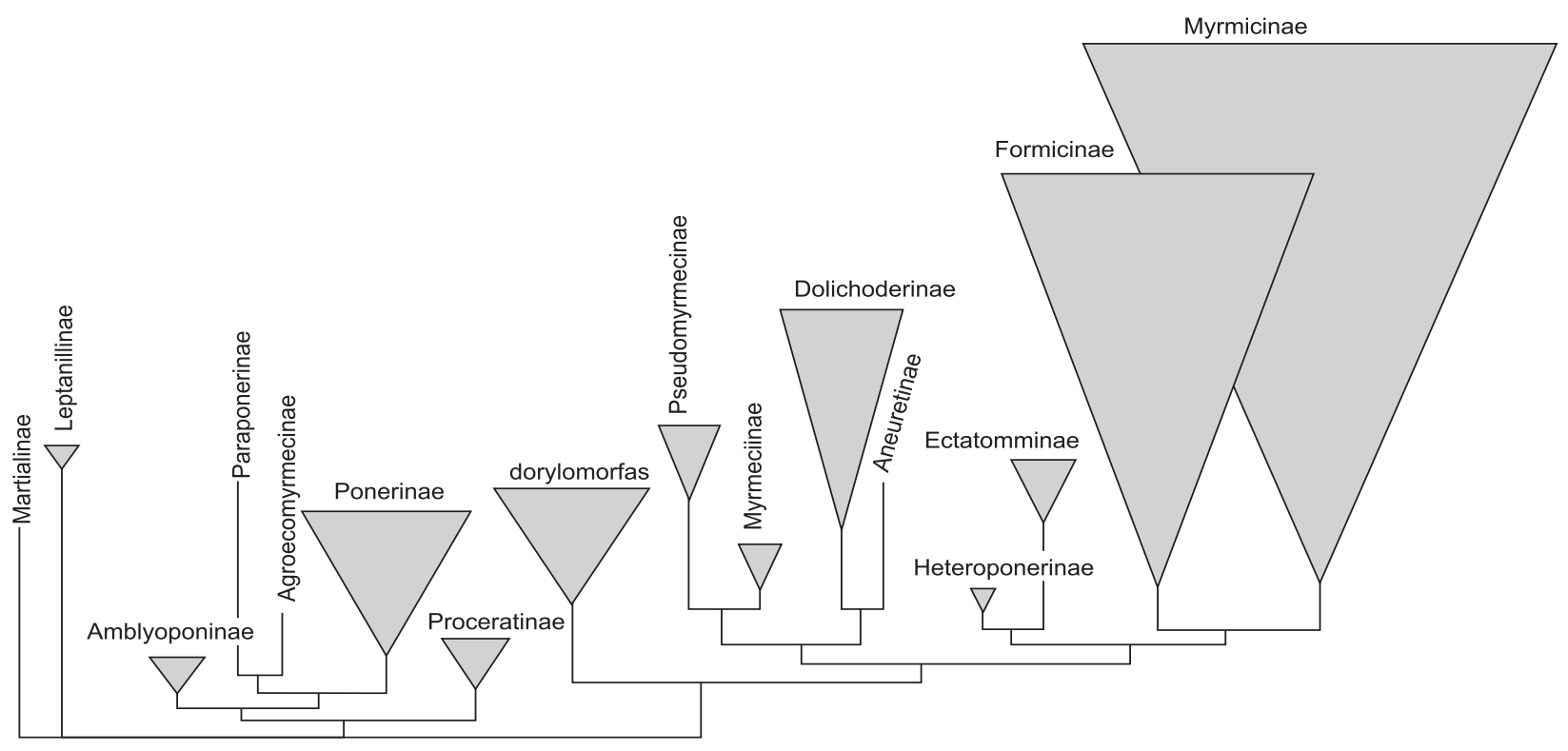


Discothyrea que aparece como grupo-irmão de um clado formado por Probolomyrmex + Proceratium. Da mesma forma, as topologias obtidas por Keller (2011) com base em caracteres morfológicos apontam para a mesma relação interna, o que pode ser considerada uma boa indicação de que esta possa ser de fato a história evolutiva do grupo. Ainda assim, um estudo concentrado na subfamília e que inclua um número maior de espécies se faz urgentemente necessário para uma melhor compreensão das relações filogenéticas em Proceratiinae.

Pelo conceito atual de subfamílias de formigas, Proceratiinae ainda engloba as duas tribos e três gêneros nela combinados por Bolton (2003): Discothyrea e Proceratium em Proceratiini e Probolomyrmex em Probolomyrmecini. A subfamília está presente em todo o mundo, especialmente nas regiões tropicais, onde a maior parte de suas cerca de 140 espécies ocorrem.

Nas Américas, Proceratiinae apresenta cerca de 40 espécies distribuídas desde o sul do Canadá até a Argentina. No Brasil, os três gêneros da subfamília estão representados por apenas seis espécies formalmente descritas, sendo que Probolomyrmex apresenta três espécies, Discothyrea possui duas espécies e Proceratium está representado no país por uma única espécie.

Proceratiinae pode ser morfologicamente reconhecida pela seguinte combinação de caracteres: tamanho relativamente pequeno; inserções antenais total ou parcialmente expostas; olhos reduzidos a ausentes; sutura promesonotal fundida ou ausente; garras tarsais anteriores simples, nunca pectinadas ou com dentes pré-apicais; pecíolo sem tergitos laterais; e lobo jugal ausente nas asas anteriores das formas sexuadas.

Com relação à taxonomia alfa da subfamília, até o momento, com exceção das Discothyrea neotropicais, todos os gêneros proceratiíneos foram objeto de revisões taxonômicas. Contudo, estes trabalhos encontram-se relativamente defasados e o acúmulo em coleções mirmecológicas de exemplares que não se enquadram nas descrições de espécies descritas evidencia a necessidade de novos trabalhos. As chaves taxonômicas atualmente utilizadas para a identificação de espécies neotropicais de Proceratiinae são basicamente as encontradas nos trabalhos de O'Keefe; Agosti (1997), para Probolomyrmex e Baroni-Urbani; de Andrade (2003), para Proceratium. Não menos importante é a contribuição recente de Sosa-Calvo; Longino (2008) que revisaram o estado atual de conhecimento taxonômico em Proceratiinae e forneceram chaves de identificação para as espécies da subfamília, com ênfase nos táxons que ocorrem na Colômbia.

A subfamília é relativamente pouco representada em coleções mirmecológicas. Isso ocorre porque os indivíduos são raramente capturados com as técnicas mais tradicionais de coleta. $\mathrm{O}$ extrator de Winkler tem se mostrado o método mais eficiente para amostrar exemplares de Proceratiinae, embora estes sejam coletados sempre em número bastante reduzido. Esta aparente raridade se deve em grande parte ao hábito críptico das espécies e às baixas densidades populacionais das colônias, que raramente alcançam 100 indivíduos. Os ninhos são normalmente encontrados em cavidades no solo de florestas, entre as folhas da serapilheira, troncos em decomposição e sob rochas.

Com relação ao hábito alimentar, as observações feitas até então sugerem uma preferência destas formigas por predar ovos de pequenos artrópodes de solo, em especial aracnídeos (BROWN, 1954; 1979). Virtualmente nada é conhecido sobre o sistema de forrageamento das espécies proceratiíneas e suas estratégias reprodutivas.

\section{Representantes de Proceratiinae no Brasil}

\section{Discothyrea Roger, 1863}

Discothyrea é representado atualmente por 32 espécies válidas distribuídas mundialmente. São formigas diminutas reconhecidas principalmente pela seguinte combinação de caracteres: corpo compacto e de perfil arredondado; mandíbulas sem dentes ou dentículos nas margens mastigatórias; inserções antenais posicionadas sobre uma plataforma fronto-clipeal que se estende anteriormente na cápsula cefálica cobrindo as mandíbulas em vista frontal; segmento apical das antenas formando uma clava extremamente desenvolvida; escapos antenais curtos, nunca ultrapassando a margem posterior da cabeça; olhos fortemente reduzidos, formados por um único omatídeo ou completamente ausentes; e quarto segmento abdominal (segundo gastral) fortemente curvado para frente, projetando-se ventralmente sob o mesossoma (Figura 7.3).

Na Região Neotropical, o gênero é representado por oito espécies, sendo que apenas duas delas 
Figura 7.3 - Discothyrea sexarticulata, operária em (a) vista frontal e (b) vista lateral. Foto: Estella Ortega (Antweb, CASENT0281862).

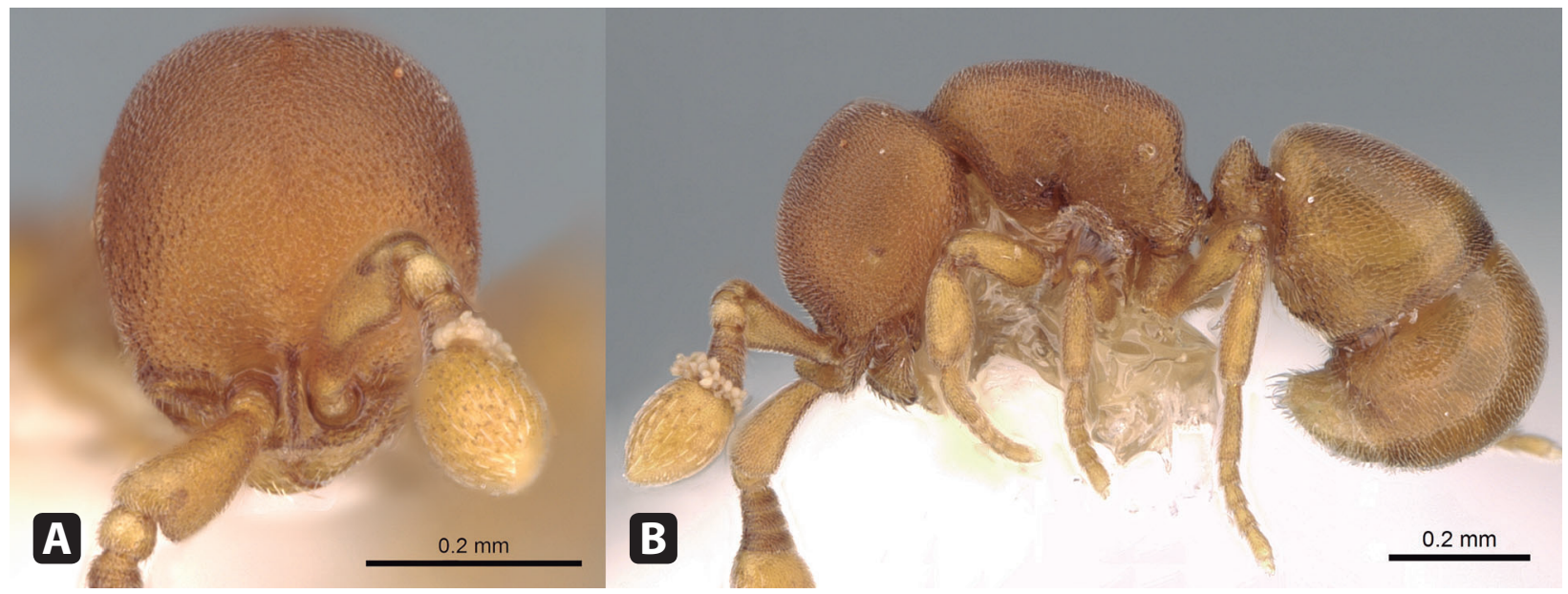

estão registradas para o Brasil, D. neotropica Bruch e D. sexarticulata Borgmeier. Tradicionalmente, os exemplares deste gênero coletados no Brasil são identificados com base no número de segmentos antenais (seis em $D$. sexarticulata e nove em $D$. neotropica). Isto ocorre como consequência dos trabalhos de Borgmeier (1949; 1954) que utilizou esta característica como critério para separar as espécies neotropicais de Discothyrea nos últimos estudos taxonômicos conduzidos para o gênero nesta região. Contudo, este caráter utilizado isoladamente tem se mostrado frágil na delimitação de espécies do gênero (SOSA-CALVO; LONGINO, 2008).

Outro caráter de importância taxonômica é a posição dos espiráculos propodeais, que também apresenta graus significativos de variação. Devido ao tamanho reduzido das espécies do gênero, a tarefa de se conferir nomes aos indivíduos coletados pode consistir em um desafio considerável, já que as dimensões dos exemplares tornam muito difíceis a contagem de segmentos antenais e a observação de demais estruturas. De fato, o pequeno número de espécies reportado para o Brasil é provavelmente um artefato da ausência de trabalhos taxonômicos para o grupo nesta região. Assim, dada a natureza problemática dos limites taxonômicos entre espécies de Discothyrea, é muito provável que um estudo minucioso revele novas espécies para o Brasil. Felizmente, neste momento há um estudo em andamento que almeja a revisão taxonômica das espécies de Discothyrea do Novo Mundo (J. SOSA-CALVO, em prep.).

Como já mencionado para a subfamília, a coleta de exemplares de Discothyrea é um evento incomum comparado à frequência com que grande parte dos gêneros poneroides é registrada. Estas formigas possuem hábitos crípticos e colônias de tamanho populacional reduzido. Os ninhos são encontrados nos interstícios da serapilheira e em troncos em decomposição no solo de florestas.

As espécies deste gênero que possuem sua biologia conhecida são conhecidas por estocar e predar ovos de aracnídeos (BROWN, 1954; 1979). A especialização neste item alimentar é tão significativa que algumas espécies não apenas consomem ovos de aranhas, mas também podem estabelecer suas colônias no interior das ootecas destes animais (DEJEAN et al., 1999).

\section{Probolomyrmex Mayr, 1901}

Probolomyrmex é atualmente representado por 21 espécies distribuídas em todas as regiões tropicais do mundo. Este é o único gênero incluído na tribo Probolomyrmecini (BOLTON, 2003) e pode ser morfologicamente diagnosticado pelos seguintes caracteres: corpo alongado e filiforme; mandíbulas com seis a oito dentes; inserções antenais posicionadas sobre uma plataforma frontoclipeal que se estende anteriormente na cápsula cefálica cobrindo as mandíbulas em vista frontal; antenas com 12 segmentos; olhos ausentes; quarto segmento abdominal (segundo gastral) nunca curvado para frente (Figura 7.4). De fato, o corpo alongado e a ausência de uma curvatura ventral no segundo segmento do gáster são suficientes para separar Probolomyrmex dos demais proceratíneos.

Taylor (1965) foi o primeiro a apresentar uma revisão global do gênero com base em um 
Figura 7.4 - Probolomyrmex petiolatus, operária em (a) vista frontal e (b) vista lateral. Foto: April Nobile (Antweb, CASENT 0102222).

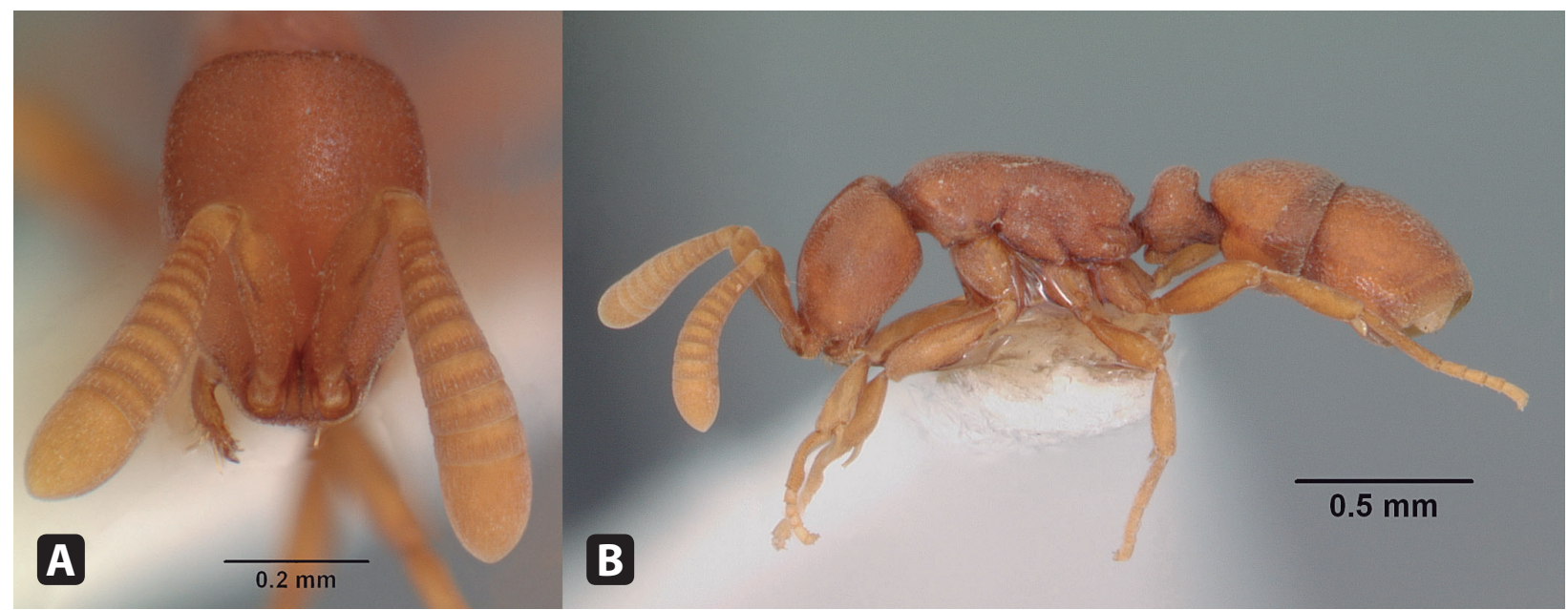

número relativamente pequeno de indivíduos; tanto que neste trabalho a Região Neotropical foi representada por espécimes de apenas duas localidades. Em 1994, Agosti revisou taxonomicamente as espécies de Probolomyrmex do Novo Mundo e forneceu uma chave de identificação para as espécies até então conhecidas. Esta chave foi atualizada três anos depois com a descrição de uma nova espécie para a Costa Rica (O'KEEFE; AGOSTI, 1997) e segue sendo a principal referência para a identificação das espécies neotropicais do gênero. Atualmente são conhecidas quatro espécies de Probolomyrmex para os neotrópicos: P. boliviensis Mann, P. brujitae Agosti, P. petiolatus Weber e P. guanacastensis O'Keefe; Agosti.

$O$ primeiro registro do gênero para o Brasil foi formalmente publicado por Delabie et al. (2001) que relatam a presença de $P$. petiolatus para uma área da Amazônia Oriental. Desde então duas outras espécies foram reportadas para o Brasil, P. brujitae (NASCIMENTO et al., 2004) e P. boliviensis (R.M. FEITOSA, dados não publicados). Assim, com exceção de P. guanacastensis, restrita à Costa Rica, todas as demais espécies do gênero ocorrem no Brasil, distribuídas em todas as regiões do país, com exceção da Região Sul.

Embora amplamente distribuído e sendo o gênero de Proceratiinae com o maior número de espécies registradas no Brasil, Probolomyrmex está longe de ser considerada uma formiga comumente coletada. Como já mencionado para Proceratiinae, a coleta de exemplares de Probolomyrmex é um evento bastante incomum. Com a popularização do extrator de Winkler como técnica de coleta de formigas, o número de exemplares em coleções mirmecológicas tem aumentado substancialmente. Este aumento na representatividade do gênero em coleções brasileiras tem evidenciado o quão frágeis parecem ser os limites morfológicos entre as espécies do gênero. Ainda, entre o material recém-acumulado, certamente existem espécies ainda não descritas e que devem aumentar a diversidade do gênero para a Região Neotropical. As espécies brasileiras do gênero são objeto de uma revisão taxonômica em andamento (R.M. FEITOSA, em prep.).

A dificuldade em se coletar exemplares de Probolomyrmex está diretamente relacionada ao hábito de vida críptico das espécies dos gêneros. Os ninhos são feitos no solo, entre folhas na serapilheira e, especialmente, em cavidades no interior de frutos secos, troncos e gravetos caídos no solo de florestas úmidas. O corpo filiforme e a ausência de olhos indicam que as formigas deste gênero raramente se expõem na superfície do solo e que preferem os interstícios da serapilheira e suas cavidades naturais.

Dada a similaridade morfológica com os demais gêneros da subfamília, Taylor (1965) sugere que os membros de Probolomyrmex sejam predadores especialistas de ovos de aracnídeos, assim como Discothyrea e Proceratium. No entanto, Ito (1998) observou a predação de miriápodes polixenídeos por $P$. dammermani, indicando que a dieta das espécies de Probolomyrmex pode ser mais ampla do que a dos demais gêneros.

\section{Proceratium Roger, 1863}

Entre os gêneros de Proceratiinae, Proceratium é o que apresenta melhor situação taxonômica. Isso se 
deve ao monumental trabalho de Baroni-Urbani e de Andrade (2003), que revisaram o gênero globalmente. Além de ilustrar e lidar com a taxonomia das espécies viventes e fósseis de todo o mundo, os autores apresentam uma análise cladística para o gênero e fornecem chaves de identificação que seguramente são as principais ferramentas atuais para se nomear espécies de Proceratium em todas as regiões biogeográficas.

Com 77 espécies válidas, Proceratium é o gênero mais diverso da subfamília e se encontra distribuído nas regiões tropicais e temperadas do planeta. Este gênero compõe a tribo Proceratiini ao lado de Discothyrea e compartilha com este a forte curvatura ventral do segundo segmento gastral. Contudo, Proceratium pode ser rapidamente separado dos demais gêneros da subfamília por não apresentar as inserções antenais sobre uma plataforma fronto-clipeal, de modo que as mandíbulas são perfeitamente visíveis em vista frontal (Figura 7.5).

No Novo Mundo são conhecidas 23 espécies viventes de Proceratium, o que demonstra que o gênero também é atualmente o mais diverso da subfamília neste continente. Apesar da grande diversidade global, o Brasil registra uma única espécie para o gênero, $P$. brasiliense, encontrada em áreas de Floresta Atlântica do sul da Bahia à Santa Catarina.

Entre os representantes de Proceratiinae no Brasil, Proceratium é sem dúvida o mais raramente coletado. Operárias são eventualmente capturadas sozinhas ou em pares em amostras de serapilheira, o que faz com que a representatividade do gênero em coleções mirmecológicas brasileiras seja muito baixa. Como já mencionado para os demais membros da subfamília, as colônias de Proceratium são estabelecidas na serapilheira e em madeira em decomposição no solo de florestas.

A biologia de Proceratium é de certa forma similar à de Discothyrea. Brown $(1954 ; 1979)$ conduziu alguns experimentos em condições artificiais e demonstrou que operárias de Proceratium preferem ovos de aranhas a fragmentos de insetos como recurso alimentar.

\section{Perspectivas}

Como visto, talvez com exceção de Proceratium, os demais gêneros de Proceratiinae necessitam com relativa urgência de uma atualização taxonômica, especialmente nos neotrópicos. Por suas dimensões continentais e heterogeneidade de ecossistemas, o Brasil certamente guarda uma porção significativa da diversidade de formigas desta subfamília que ainda aguarda descrição formal. O emprego relativamente recente de técnicas massivas de coleta de formigas de serapilheira, aplicadas principalmente em levantamentos quantitativos, tem proporcionado um aumento substancial da representatividade de formigas, até então tidas como raras, em coleções entomológicas. Este material é com certeza o substrato sobre o qual novas propostas taxonômicas poderão ser desenvolvidas.

Em Proceratiinae, dois trabalhos em andamento aparecem, ao lado da publicação de Baroni-Urbani; de Andrade (2003), como as futuras

Figura 7.5 - Proceratium catio, operária em (a) vista frontal e (b) vista lateral. Foto: April Nobile (Antweb, CASENT 01178756).

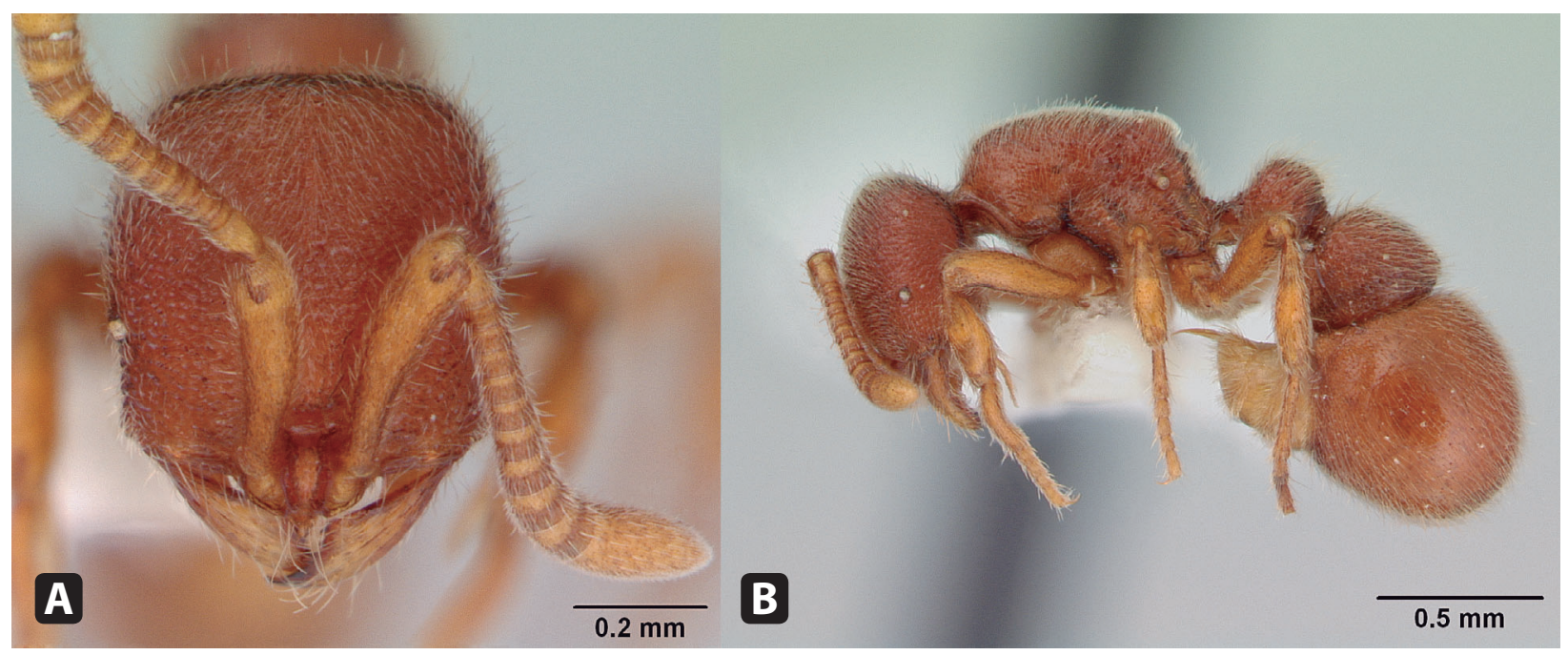


ferramentas de identificação para as espécies da subfamília com ocorrência no Brasil. Tratam-se das revisões taxonômicas de Discothyrea (J. SOSA-CALVO, em prep.) e Probolomyrmex (R.M. FEITOSA, em prep.) para a Região Neotropical e para o Brasil, respectivamente. Com a publicação destes trabalhos, pesquisadores brasileiros serão, esperançosamente, capazes de identificar todas as espécies de Proceratiinae com ocorrência no país.

\section{Referências}

BOLTON, B. Identification guide to the ant genera of the world. Harvard University Press. Cambridge, Massachusetts, 1994.222 p.

BARONI-URBANI, C.; DE ANDRADE, M. L. The ant genus Proceratium in the extant and fossil record (Hymenoptera: Formicidae). Museo Regionale di Scienze Naturali Monografie (Turin), v. 36, p. 1-492, 2003.

BOLTON, B. A New General catalogue of the Ants of the Word. Harvard University Press. Cambridge, Massachusetts. 1995.504 p.

BOLTON, B. Synopsis and classification of Formicidae. Memoirs of the American Entomological Institute, v. 71, p. 1-370, 2003.

BORGMEIER T. Formigas novas ou pouco conhecidas de Costa Rica e da Argentina (Hymenoptera: Formicidae). Revista Brasileira de Biología, v. 9, p. 201- 210, 1949.

BORGMEIER T. Uma nova Discothyrea com seis articulos antenais (Hymenoptera: Formicidae). Revista Brasileira de Entomologia, v. 1, p. 191-194. 1954.

BRADY, S. G.; SCHULTZ, T. R.; FISHER, B. L.; WARD, P. S. Evaluating alternative hypotheses for the early evolution and diversification of ants. Proceedings of the National Academy of Sciences of the United States of America, v. 13(48), p. 18172-18177, 2006.

BROWN, W. L., Jr. Predation of arthropod eggs by the ant genera Proceratium and Discothyrea. Psyche, v. 64, p. 115, 1954.

BROWN, W. L., Jr. Contributions toward a reclassification of the Formicidae. II. Tribe Ectatommini (Hymenoptera). Bulletin of the Museum of Comparative Zoology of Harvard College, v. 118, p. 175-362, 1958.

BROWN, W. L., Jr. Contributions toward a reclassification of the Formicidae. V. Ponerinae, tribes Platythyreini, Cerapachyini, Cylindromyrmecini, Acanthostichini, and Aenictogitini. Search: Agriculture, Cornell University Agricultural Experiment Station, v. 5, p. 1-115, 1975.
BROWN, W. L., Jr. A remarkable new species of Proceratium with dietary and other notes on the genus (Hymenoptera: Formicidae). Psyche, v. 86, p. 337-346, 1979.

DEJEAN, A.; GRIMAL A.; MARHERBE M. C.; SUZZONI J. P. From specialization in spider egg predation to an original nesting mode in a "primitive" ant: a new kind of leptobiosis. Naturwissenschaften, $v$. 86, p. 133-137, 1999.

DELABIE, J. H. C., VASCONCELOS, H. L., VILHENA, J. M. S; AGOSTI, D. First record of the ant genus Probolomyrmex (Hymenoptera: Formicidae) in Brazil. Revista de Biologia Tropical, v. 49, p. 397-398, 2001.

FEITOSA, R. S. M. Revisão Taxonômica e Análise Filogenética de Heteroponerinae (Hymenoptera: Formicidae). Tese de Doutorado apresentada ao Programa de Pós-Graduação em Entomologia da Faculdade de Filosofia Ciências e Letras da Universidade de São Paulo. Ribeirão Preto. São Paulo, Brasil. 2011. XIII + 297 p.

ITO, F. Colony composition and specialized predation on millipedes in the enigmatic ponerine ant genus Probolomyrmex (Hymenoptera, Formicidae). Insectes Sociaux, v. 45, p. 79-83. 1998.

KELLER, R. A. Cladistics of the tribe Ectatommini (Hymenoptera: Formicidae): a reappraisal. Insect Systematics; Evolution, v. 31, p. 59-69, 2000.

KELLER, R. A. A phylogenetic analysis of ant morphology (Hymenoptera: Formicidae) with special reference to the poneromorph subfamilies. Bulletin of the American Museum of Natural History, v. 355, p. $1-90,2011$.

LATTKE, J. E. Phylogenetic relationships and classification of ectatommine ants (Hymenoptera: Formicidae). Entomologica Scandinavica, v. 25, p. 105-119, 1994.

MOREAU, C. S.; BELL, C. D.; VILA, R.; ARCHIBALD, B.; PIERCE, N. E. Phylogeny of the ants: diversification in the age of angiosperms. Science, v. 312, p. 100-104. 2006.

NASCIMENTO, I. C.; DELABIE, J. H. C.; CAMPIOLO, S.; MARIANO NETO, E. Présence de Probolomyrmex brujitae Agosti, 1994, au Brésil (Hym., Formicidae). Bulletin de la Société Entomologique de France, v. 109, p. 321-322. 2004.

O'KEEFE, S. T.; AGOSTI D. A new species of Probolomyrmex (Hymenoptera: Formicidae) from Guanacaste, Costa Rica. Journal of the New York Entomological Society, v. 105, p. 190-192. 1997. 
OUELLETTE, G. D.; FISHER, B. L.; GIRMAN, D. J. Molecular systematics of basal subfamilies of ants using $28 \mathrm{~S}$ rRNA (Hymenoptera: Formicidae). Molecular Phylogenetics and Evolution, v. 40, p. 359369. 2006.

RABELING, C.; BROWN J. M.; VERHAAGH, M.

Newly discovered sister lineage sheds light on early ant evolution. Proceedings of the National Academy of Science, v. 105 (39), p. 14913-14917. 2008.
SOSA-CALVO, J.; LONGINO, J. T. Subfamilia Proceratiinae. In: JIMÉNEZ, E.; FERNÁNDEZ, F.; ARIAS, T. M.; LOZANO-ZAMBRANO, F. H. (Org.). Sistemática, biogeografía y conservación de las hormigas cazadoras de Colombia. Instituto de Investigación de Recursos Biológicos Alexander von Humboldt. Bogotá. 2008. p. 219-237.

TAYLOR, R. A monographic revision of the rare tropicopolitan ant genus Probolomyrmex Mayr (Hymenoptera: Formicidae). Transactions of the Royal Entomological Society of London, v. 117, p. 345-365. 1965. 